\title{
Revista Brasileira \\ de Enfermagem Necessidades de cuidados de enfermagem em RFRF Terapia Intensiva: evolução diária dos pacientes segundo o Nursing Activities Score (NAS)
}

\author{
The need for nursing care in Intensive Care Units: daily patient \\ assessment according to the Nursing Activities Score (NAS)
}

Necesidades de cuidados de enfermería en la Unidad de Cuidados Intensivos: evaluación diaria de los pacientes según el Nursing Activities Score (NAS)

Leilane Andrade Gonçalves

Enfermeiro assistencial da UTI adulto do Hospital Universitário da USP.

Paulo Carlos Garcia

Enfermeiro assistencial da UTI adulto do Hospital Universitário da USP.

Maria Cecília Toffoleto

Mestre em enfermagem pela Escola de Enfermagem da USP.

Sandra Cristina Ribeiro Telles

Mestre em enfermagem pela Escola de Enfermagem da USP.

Kátia Grillo Padilha

Enfermeiro. Professora Associada do Departamento de Enfermagem MédicoCirúrgica da Escola de Enfermagem da USP.

Submissão: 02/02/2005

Aprovação: 02/10/2005

\section{RESUMO}

O estudo teve como objetivos caracterizar os pacientes internados na UTI quanto aos dados bio-sociais e de internação, e verificar as necessidades diárias de cuidados de enfermagem, segundo o NAS. A amostra foi constituída por 50 pacientes adultos admitidos consecutivamente na UTI de um Hospital Universitário do município de São Paulo. Concluiu-se que a maioria dos pacientes tinha idade acima de 60 anos, permaneceu, em média, 3,5 dias na UTI, foi procedente do Pronto Socorro e recebeu alta para a Unidade Semi-Intensiva. A média do NAS foi de $66,5 \%( \pm 9,1)$, constatando-se que permaneceu acima de $50,0 \%$ no decorrer de toda internação na UTI.

Descritores: Carga de trabalho; Cuidados intensivos; Enfermagem.

\section{ABSTRACT}

This study aimed to characterize patients admitted to ICU according to their bio-social factors and admissions and verify the daily needs for nursing care according to NAS. The sample was composed by 50 adult patients who were consecutively admitted to the ICU of a University Hospital in São Paulo city. It was concluded that most patients were older than 60 with an average of 3.5-day stay in the ICU; they came from the Emergency Room and were referred to the Semi-Intensive Care Unit. NAS average was $66,5 \%( \pm 9,1)$ with a score higher than $50.0 \%$ during their stay in the ICU.

Descriptors: Workload; Critical care; Nursing.

\section{RESUMEN}

El trabajo tuvo como objetivos caracterizar a los pacientes internados en la UCl en función de los datos biosociales y de internación; verificar las necesidades diarias de cuidados de enfermería según el NAS. La muestra estuvo constituida por 50 pacientes adultos admitidos consecutivamente en la UCI de un Hospital Universitario de la municipalidad de Sao Paulo. Se concluyó que la mayoría de los pacientes tenía edad superior a los 60 años; permaneció, en promedio 3,5 días en la UCl, procedía de Emergencia y fue dado de alta para la Unidad Semi-Intensiva. El promedio del NAS fue de 66,5\% $( \pm 9,1)$, constatándose que permaneció por encima del $50,0 \%$ en el transcurso de toda la internación en la UCl.

Descritores: Carga de trabajo; Cuidados críticos; Enfermería.

Gonçalves LA, Garcia PC, Toffoleto MC, Telles SCR, Padilha KG. Necessidades de cuidados de enfermagem em terapia intensiva: evolução diária dos pacientes segundo o Nursing Activities Score (NAS). Rev Bras Enferm 2006 jan-fev; 59(1): 56-60.

\section{INTRODUÇÃO}

A necessidade de avaliar objetivamente quem são os pacientes graves que requerem tratamento intensivo tem tornado a utilização de instrumentos de medida de gravidade, prática indispensável nas UTIs, face aos altos custos dessas unidades.

No entanto, tão importante quanto avaliar a gravidade e predizer mortalidade dos pacientes admitidos na UTI, a avaliação das necessidades de cuidados e, conseqüentemente, da demanda de trabalho de enfermagem, tem sido relevante quando se busca aliar qualidade da assistência, otimização de recursos e redução de custos. Assim, tais índices são cada vez mais necessários como ferramenta assistencial e administrativa voltada para a adequação de recursos materiais, equipamentos e de pessoal de enfermagem na UTI(1).

Um dos instrumentos desenvolvidos com essa finalidade foi o Therapeutic Intervention Scoring System (TISS), que desde a sua criação em 1974, teve como propósito medir a gravidade do paciente segundo a complexidade e o grau de invasividade das intervenções, bem como o tempo 
dispensado pela enfermagem para a realização de determinados procedimentos $^{(2,3)}$.

Porém, com o desenvolvimento de índices de gravidade de base fisiológica ${ }^{(4-6)}$, o TISS, após várias versões ${ }^{(3,7,8)}$, foi reestruturado e mais direcionado para a avaliar as necessidades de cuidados e carga de trabalho de enfermagem na UTI, passando a denominar-se Nursing Activities Score (NAS) ${ }^{(9)}$. Incorporando atividades de enfermagem não contempladas nas versões anteriores, como procedimentos de higiene, suporte e cuidados aos familiares/pacientes, tarefas administrativas e gerenciais, o escore NAS expressa a porcentagem de tempo gasto por um profissional de enfermagem na assistência direta ao doente crítico na UTI, em 24 horas $^{(10)}$.

Apesar da inquestionável necessidade de se estimar carga de trabalho de enfermagem em UTI com o uso de instrumentos de medida específicos para os doentes críticos, ainda são poucos os estudos existentes no nosso meio, razão pela qual julgou-se de interesse a presente investigação. Além disso, há que se ressaltar que a disponibilidade do NAS recentemente traduzido e validado para a realidade brasileira ${ }^{(9)}$, tornou possível a sua aplicação prática em nosso meio.

Constituem objetivos do estudo: caracterizar os pacientes adultos internados na UTI, segundo o NAS; descrever a demanda de trabalho de enfermagem no decorrer da internação na UTI; e verificar a influência das variáveis idade, tempo de permanência, procedência e destino após a saída da unidade na carga de trabalho de enfermagem.

\section{CASUÍSTICA E MÉTODO}

O estudo, de abordagem quantitativa, foi desenvolvido na Unidade de Terapia Intensiva (UTI) de adultos de um Hospital Universitário do município de São Paulo, instituição de atendimento secundário, que dispõe de um total de 12 leitos de UTI e 9 destinados à Unidade SemiIntensiva (USI).

A população foi constituída por 50 pacientes com idade igual ou maior do que 18 anos, admitidos consecutivamente na UTI, a partir de 26 de setembro de 2002, e que nela permaneceram por um período mínimo de 24 horas. Readmissões foram excluídas do estudo.

Para a coleta de dados utilizou-se um impresso denominado "Avaliação das necessidades diárias de cuidado", composto de 3 partes: identificação do paciente, dados clínicos e NAS, com a discriminação dos seus itens componentes.

Após a aprovação do projeto pela Comissão de Ética em Pesquisa do hospital, campo deste estudo, foi feito o acompanhamento prospectivo dos pacientes, da internação à alta da UTI, até a saída do último paciente da amostra, o que ocorreu no dia 03 de novembro de
2002. Atendido o critério de inclusão e com o Termo de Consentimento Livre e Esclarecido assinado pelo paciente ou seu responsável legal, procedeu-se o preenchimento do NAS, tendo por base dados do prontuário e ficha de controle dos pacientes, acrescidos de informações complementares fornecidas pelos enfermeiros, sempre que necessário. Para fins de padronização, foram consideradas as informações referentes às 24 horas do dia anterior que se completavam às 8 horas da manhã. Quanto ao primeiro dia de internação, foram computadas as intervenções realizadas da hora da admissão na UTI até às 8 horas do dia seguinte, independente de completarem ou não 24 horas. Também no dia da alta, além dos dados registrados às 8 horas da manhã, foram computados aqueles relacionados ao período compreendido entre às 8 horas e o momento de saída.

Os dados foram armazenados em um banco eletrônico criado no programa Excel 2000-Windows 98 e as análises estatísticas realizadas no programa SPSS 10.0. Os resultados referentes às características demográficas foram submetidos à análise descritiva.

Para o estudo da associação da carga de trabalho de enfermagem e as variáveis idade e destino após a alta da UTI foi aplicado o teste não paramétrico de Mann-Whitney, enquanto que a análise da relação da carga de trabalho com as variáveis tempo de internação na UTI e procedência dos pacientes foi feita com a utilização do teste não paramétrico de Kruskal-Wallis. Foram considerados estatisticamente significantes os resultados cujos valores de $p$ foram inferiores a 0,05 .

\section{RESULTADOS}

\subsection{Caracterização dos dados demográficos da amostra}

Do total de 50 pacientes que compuseram o estudo foram obtidas 339 medidas do escore NAS.

Com relação às características demográficas da amostra, houve igual distribuição de pacientes do sexo masculino e feminino, ambos com $50,0 \%$. A média de idade foi de 70 anos $( \pm 15,9)$, com variação entre 20 e 91 anos. Pacientes com idade acima de 60 anos foram a maioria $(64,0 \%)$, seguidos de $36,0 \%$ com idade entre 20 e 60 anos. Verificou-se que $84,0 \%$ dos pacientes eram portadores de doença crônica pré-existente, dos quais $61,9 \%, 35,7 \%$ e $2,4 \%$ apresentaram, respectivamente 1,2 e 3 doenças.

Com relação aos dados da internação (Tabela 1), 40,0\% dos pacientes foram provenientes do Pronto Socorro (PS), $24,0 \%$ da USI e $20,0 \%$ do Centro Cirúrgico (CC). Dos $22,0 \%$ submetidos à cirurgia, apenas $2(9,1 \%)$ foram submetidos à cirurgia eletiva. Internações por motivos clínicos $(78,0 \%)$ e doenças do sistema cardiovascular $(70,0 \%)$ foram predominantes na amostra. A média de permanência foi de 3,5

Tabela 1. Número e porcentagem de pacientes internados na UTI, segundo variáveis de internação. São Paulo, 2002.

\begin{tabular}{|c|c|c|}
\hline CARACTERISTICAS & $\mathbf{N}$ & $\%$ \\
\hline \multicolumn{3}{|l|}{ Procedência } \\
\hline Pronto Socorro & 20 & 40,0 \\
\hline Unidade Semi-Intensiva & 12 & 24,0 \\
\hline Centro Cirúrgico & 10 & 20,0 \\
\hline Unidade de Internação & 7 & 14,0 \\
\hline Centro Obstétrico & 1 & 2,0 \\
\hline \multicolumn{3}{|l|}{ Tipo de Internação } \\
\hline Clínica & 39 & 78,0 \\
\hline Cirúrgica-urgência & 10 & 20,0 \\
\hline Cirúrgica-eletiva & 1 & 2,0 \\
\hline \multicolumn{3}{|c|}{ Tempo de Permanência (em dias) } \\
\hline 1 a 5 & 32 & 64,0 \\
\hline 6 a 10 & 9 & 18,0 \\
\hline$>10$ & 9 & 18,0 \\
\hline \multicolumn{3}{|l|}{ Destino após a saída da UTI } \\
\hline Semi-intensiva & 27 & 54,0 \\
\hline Óbito & 19 & 38,0 \\
\hline Unidade de Internação & 4 & 8,0 \\
\hline
\end{tabular}


dias, variação de 1 a 26 dias. A maioria dos pacientes (64,0\%) permaneceu na UTI por um período de 1 a 5 dias. Igual porcentagem $(18,0 \%)$ esteve internada de 6 a 10 e mais do que 10 dias. Referente ao destino após a saída da UTI, enquanto que $54,0 \%$ dos pacientes foram transferidos para a USI, 38,0\% foram a óbito.

3.2 Caracterização das necessidades de cuidados de enfermagem e demanda de trabalho de enfermagem

A análise das necessidades de cuidados e demanda de trabalho de enfermagem mostrou uma média NAS de $66,5 \%( \pm 9,1)$, mediana de $70,7 \%$, com variação de $47,6 \%$ a $82,4 \%$.

Quanto à evolução diária, vale destacar que os escores do primeiro e último dias, referem-se à média do tempo que os pacientes permaneceram na UTI no dia da admissão $(12,6 \mathrm{hs} \pm 6,1)$ e da saída da unidade $(16,0 \mathrm{hs} \pm 5,5)$.

Referente à média do NAS, verifica-se um aumento gradativo da pontuação do segundo $(67,7 \%)$ ao oitavo dia $(73,5 \%)$, quando atingiu a maior média diária. Nota-se, a partir deste dia, queda gradativa até 0 vigésimo dia $(56,2 \%)$ com 2 picos de elevação neste intervalo, isto é, um de $69,4 \%$ no décimo quarto dia e outro de $67,9 \%$ no décimo oitavo dia, permanecendo, a partir daí, com pontuação de cerca de $56,2 \%$ até o vigésimo sexto dia.

No que diz respeito ao quantitativo de pacientes por dia de internação, verifica-se queda expressiva de 50 para 32 do primeiro ao quarto dia, com diminuição para cerca de metade (14 doentes) do $4^{\circ}$ ao $8^{\circ}$ dia. A partir desse período, houve queda gradativa para 9 pacientes até $020^{\circ}$ dia de internação, com a permanência de um único paciente até $026^{\circ}$ dia. Independente, porém, do número de pacientes, a média diária do NAS permaneceu acima de $50,0 \%$ no decorrer de todo o período.

\subsection{Fatores relacionados à demanda de trabalho de enfermagem} na UTI

Os dados referentes às variáveis idade, procedência, tempo de permanência, destino do paciente após a saída da unidade e demanda de trabalho de enfermagem encontram-se apresentados a seguir pela análise dos dados. Verificou-se que não houve diferença estatisticamente significante para afirmar que a idade tem influência na carga de trabalho de enfermagem na UTI $(p=0,952)$. A média do NAS dos pacientes com idade menor e igual a 60 anos foi de 66,54 ( \pm $9,1)$, sendo de $66,59 \%( \pm 9,2)$ entre aqueles com mais de 60 anos.

Também com relação à procedência, não foram observadas diferenças na carga de trabalho de enfermagem de pacientes vindos das diferentes unidades $(p=0,508)$. Apesar disso, nota-se que pacientes transferidos do Centro-Obstétrico (CO) e Unidades de Internação (UI) apresentaram média do NAS maiores $(69,8 \%)$ do que os provenientes da USI, PS e CC, com pontuações de $66,5 \%, 66,4 \%$ e $64,1 \%$,

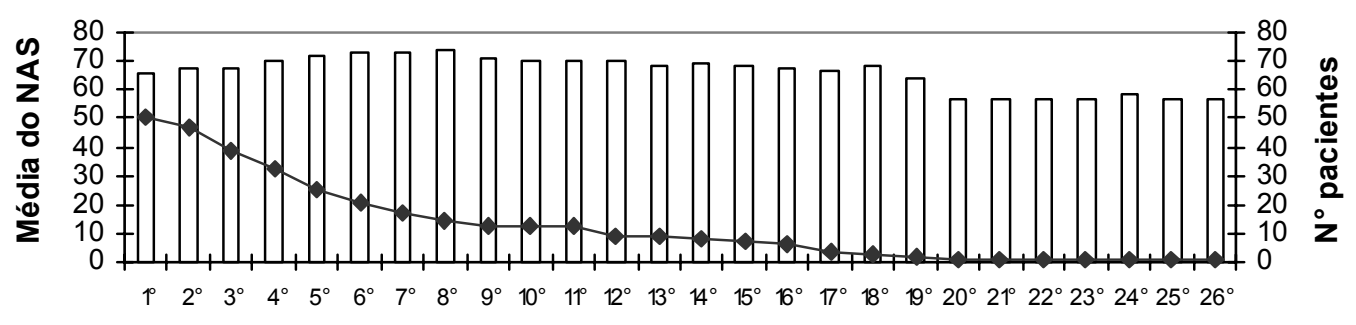

$\square$ Média NAS $\rightarrow \mathrm{N}^{\circ}$ pacientes

Dias de Internação

Figura 1. Distribuição da média diária do NAS e do número de pacientes internados na UTI, segundo dias de internação. São Paulo, 2002.

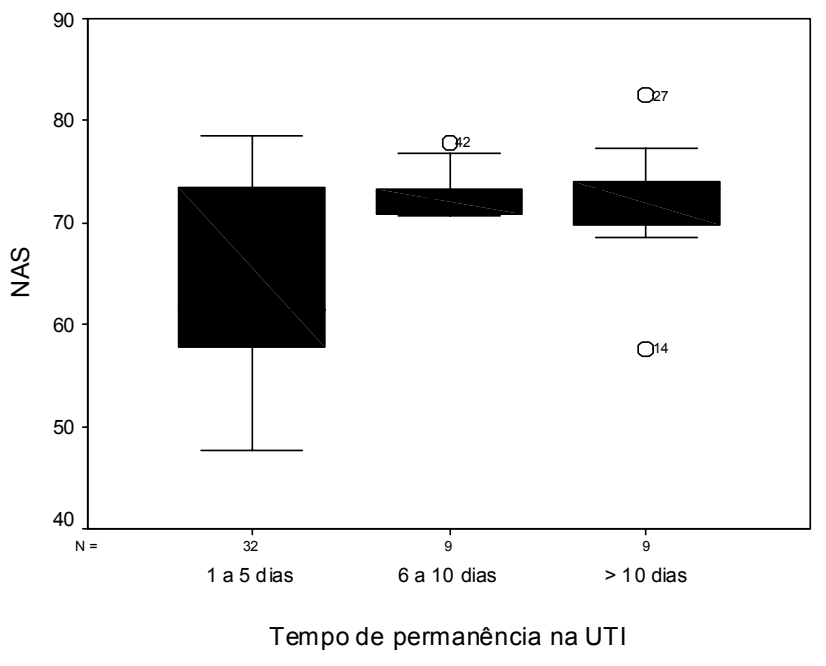

Figura 2. Box-Plot comparativo dos valores de NAS com relação ao Tempo de permanência na UTI. São Paulo, 2002. 


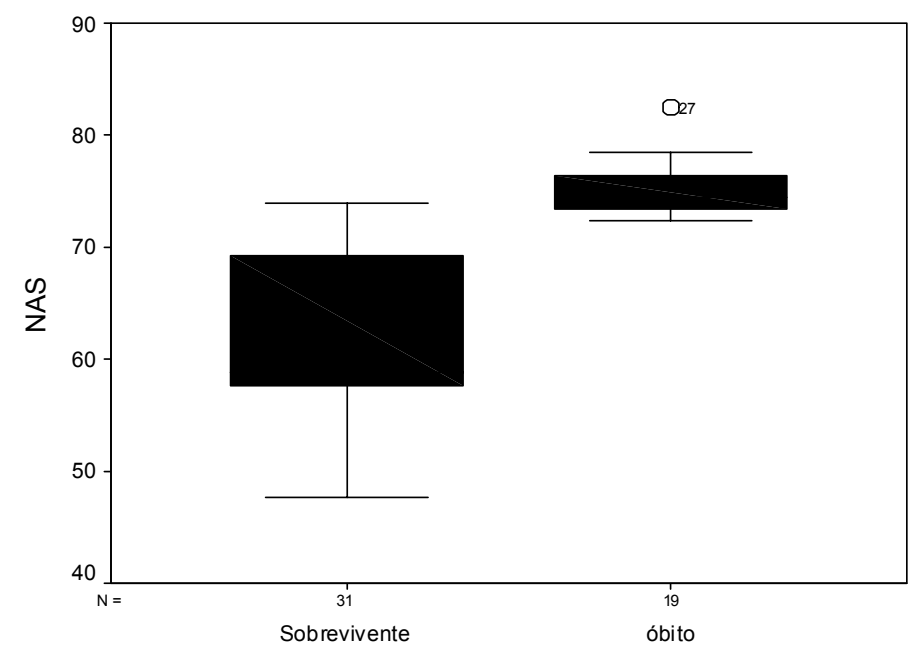

Destino

Figura 3. Box-Plot comparativo dos valores de NAS com relação ao Destino. São Paulo, 2002.

respectivamente.

No entanto, houve evidência estatística para afirmar que o tempo de permanência e o destino após a saída da unidade tiveram influência na carga de trabalho na UTI $(p=0,032)$.

Pacientes que permaneceram internados na UTI entre 6 e 10 dias e mais do que 10 dias apresentaram maiores escores, respectivamente (72,9\% e $72,1 \%)$ quando comparados com permanência menor do que 5 dias $(63,2 \%)$.

Quanto ao destino após a saída da unidade, pacientes que foram a óbito apresentaram maior média de pontuação NAS $(75,0 \%)$ do que os sobreviventes $(61,30 \%)(p<0,001)$.

\section{DISCUSSÃO}

Os resultados obtidos com relação à caracterização da amostra, mostraram que, referente a variável idade, estudos vêm apontando resultados semelhantes aos achados deste artigo ${ }^{(9,11-13)}$. Também quanto ao gênero, os resultados confirmam a literatura, pois, apesar de os estudos apontarem predominância do sexo masculino nas UTIs, observa-se uma distribuição bastante próxima do sexo feminino. Neste sentido, dois estudos nacionais que caracterizaram os pacientes de UTI, um realizado em 1990 e o outro no período de 1992-1999, mostraram a mesma distribuição entre os sexos, ambos com $57,2 \%$ de homens e $45,8 \%$ de mulheres ${ }^{(13,14)}$.

Quanto à existência de doenças crônicas prévias, a constatação de que a maioria dos doentes $(84,0 \%)$ tinha, em média, 1,4 doenças, com predomínio de alterações do sistema cardiovascular, encontra respaldo no estudo de Silva ${ }^{(15)}$, onde $70,9 \%$ dos pacientes apresentavam algum tipo de antecedente clínico relacionado a doenças do aparelho circulatório.

Sobre a procedência dos pacientes, estudos apontam que a maior parte deles é proveniente do Centro Cirúrgico (CC), seguido dos Serviços de Urgência (PS) $)^{(9,13,15)}$, diferindo, portanto, dos resultados deste estudo, que mostrou predominância dos pacientes vindos do PS $(40,0 \%)$. No entanto, considerando que grande parte dos pacientes apresentava doenças crônicas pré-existentes e que a maioria das internações ocorreu por razões clínicas $(78,0 \%)$, semelhante a outros estudos nacionais ${ }^{(9,15)}$, pode-se inferir que agravos agudos de estados mórbidos prévios, não tratados, têm levado à internação na UTI, sejam os pacientes procedentes do PS, da USI, ou da UI.
Na literatura, poucos estudos apontam a USI como sendo a unidade de procedência dos pacientes, justificada pelo fato de a maioria dos hospitais não disporem deste tipo de unidade. Porém, estudo realizado nos meses de junho, julho e agosto de 2001 na mesma UTI, campo deste estudo, cujo hospital conta com unidades de cuidados semicríticos, mostrou que 31,0\% eram procedentes dessa unidade, seguidos daqueles provenientes do PS $(28,0 \%)^{(16)}$. Diante desses resultados é de se supor que, no período de cerca de um ano entre esses estudos, houve uma maior demanda de atendimentos no PS com pacientes chegando em condições agudas com maior componente de gravidade, necessitando, portanto, de assistência intensiva.

Com relação ao tempo de permanência de um a cinco dias, predominante na amostra, os resultados são corroborados pelo Censo Brasileiro de UTIs, realizado pela Associação de Medicina Intensiva Brasileira ${ }^{(17,18)}$, onde o tempo de permanência variou de 1 a 6 dias.

A alta taxa de mortalidade encontrada, ou seja, 38,0\%, situa-se não só acima da verificada em estudos brasileiros, com variação de $29,0 \%$ a $35,0 \%(14,16,19)$, como também estrangeiros, que oscilaram entre 8,0 e $19,0 \%{ }^{(20,21)}$. Observa-se, além disso, que a taxa de mortalidade neste estudo foi maior, inclusive, daquela verificada em estudo realizado nesta mesma unidade, um ano antes, ou seja, de $33,0 \%{ }^{(16)}$. A constatação da elevada mortalidade no período analisado, talvez se justifique pela associação de diferentes fatores, entre outros, a idade avançada e doenças crônicas pré-existentes, não se podendo descartar as admissões na UTI de pacientes fora de possibilidades terapêuticas, sobretudo daqueles provenientes do PS.

Ao se investigar os escores NAS encontrados, cuja média foi de $66,5 \%( \pm 9,15)$, mediana de $70,7 \%$, portanto elevados quando se consideram as necessidades de cuidados e, conseqüentemente, a carga de trabalho de enfermagem na UTI, os resultados revelam dados importantes do ponto de vista prático. Considerando-se que o tempo gasto por um profissional de enfermagem para o cuidado de um paciente, em um plantão, é de cerca de $70,0 \%$ do seu tempo de trabalho, esses resultados trazem subsídios consistentes para a avaliação quantitativa dos recursos humanos existentes na UTI. Nesse sentido, pode-se questionar se a relação de um funcionário para dois pacientes (1:2), preconizados nas Normas e critérios para a criação das UTIs ${ }^{(22)}$ se aplicariam a essa unidade, em particular. Com base nos resultados obtidos, pode-se dizer que um profissional de enfermagem não poderia, 
sem ajuda, cuidar de 2 pacientes com a mesma pontuação durante sua jornada de trabalho, sob o risco de comprometer a sua saúde e a qualidade da assistência ao paciente.

Levando-se em conta a evolução do NAS no decorrer da internação, ou seja, do primeiro ao vigésimo sexto dia, foi possível verificar que os escores se mantiveram acima de $50,0 \%$ durante todo o período, com picos de $73,5 \%$, no oitavo dia, mostrando que pacientes com permanências elevadas na UTI continuam recebendo grandes investimentos terapêuticos, acarretando alta demanda de trabalho de enfermagem.

Nesse sentido, observou-se que pacientes com permanência acima de 6 dias apresentaram valores médios de NAS superior (cerca de $72,0 \%$ ), comparativamente àqueles com tempo de internação até cinco dias $(63,2 \%)$ com valor de $p=0,032$. Da mesma forma, pacientes que evoluíram a óbito apresentaram média NAS mais elevadas do que os que sobreviveram $(75,0 \%$ e $61,3 \%$, respectivamente) diferenças que também se mostraram estatisticamente significantes $(p<0,001)$. A alta mortalidade observada $(38,0 \%)$ e a permanência prolongada ( $>6$ dias) de $36,0 \%$ dos pacientes permitem confirmar a elevada necessidade de cuidados requeridas e a alta carga de trabalho de enfermagem nesta UTI.

Sobre a influência da idade na demanda de trabalho de enfermagem, foi interessante notar que não houve diferença entre os grupos com idade maior e menor do que 60 anos ( $p=0,952)$, ambos com média
NAS de cerca de $66,0 \%$. Pelos resultados pode-se inferir que, independente da idade, investimentos terapêuticos são aplicados no sentido de promover a melhora clínica dos pacientes.

Analisando-se a procedência, a carga de trabalho não foi diferente entre pacientes vindos das diferentes unidades hospitalares $(p=0,508)$, indicando semelhantes necessidades de cuidados de enfermagem.

Sobre a aplicação do NAS, um único trabalho foi realizado no Brasil até 0 momento, com o objetivo de traduzir e validar o instrumento para a realidade brasileira. Nele constatou-se, semelhantemente a este estudo, uma média de NAS de 67,1\%, com variação de um mínimo de $55,7 \%$ e máximo de $107,2 \%{ }^{(9)}$.

Assim, devido à inexistência de estudos internacionais com o uso do NAS, até o momento, e a localização de apenas um estudo nacional fica difícil a análise dos resultados à luz de outras realidades.

No entanto, apesar das limitações que o estudo apresenta, sobretudo pela amostra reduzida de pacientes e pela pouca experiência no uso do NAS, os resultados, obtidos por meio de medidas objetivas, mostram que os pacientes internados na UTI analisada apresentam elevada necessidade de cuidados, e conseqüentemente, alta carga de trabalho de enfermagem para os profissionais. Tais resultados, agregados a outras argumentações, que certamente o enfermeiro responsável pela Unidade é capaz de oferecer, serão de valia para a reivindicação de um quadro de pessoal justo, que promova a assistência de enfermagem que o paciente exige e que os profissionais buscam como finalidade do seu trabalho.

\section{REFERÊNCIAS}

1. Gaidzinski RR. Dimensionamento de pessoal de enfermagem em instituições hospitalares [livre-docência]. São Paulo (SP): Escola de Enfermagem, USP; 1998.

2. Cullen DJ. Therapeutic Intervention Scoring System: a method for quantitative comparison of patient care. Crit Care Med 1974; 2(2):57-60.

3. Keene AR, Cullen DJ. Therapeutic Intervention Scoring System update 1983. Crit Care Med 1983; 11(1):1-3.

4. Le Gall JR, Lemeshow S, Saulnier F. A new Simplified Acute Physiology Score (SAPS II) based on an European/ North American Multicenter Study. Jama 1993; 270(24):2957-63.

5. Knaus WA, Wagner DP, Draper EA, Zimmerrman JE. APACHE Il: a severity of disease classification system. Crit Care Med 1985; 13:818-29.

6. Lemeshow S, Teres D, Klar J, Avrunin JS, Gehlbach SH, Rapoport J. Mortality Prediction Models (MPM II) based on an Internacional cohort of intensive care unit patient. JAMA 1993; 270:2478-86.

7. Miranda DR, RijkAD, Schaufeli W. Simplified Therapeutic Intervention Scoring System: the TISS-28 itens-results from a multicenter study. Crit Care Med 1996; 24(1): 64-73.

8. Reis Miranda D, Moreno R, lapichino G. Nine Equivalents of nursing Manpower use Score (NEMS). Intens Care Med 1997; 23(7): 760-5.

9. Queijo AMG. Tradução para o português e validação de um instrumento de medida de carga de trabalho de enfermagem em unidade de terapia intensiva: Nursing Activities Score (NAS) [tese]. São Paulo (SP): Escola de Enfermagem, USP; 2002.

10. Reis Miranda D, Raoul N, Rijk A, Schaufeli W, lapichino G. Nursing Activities Score. Crit Care Med 2003; 31(2): 374-82.

11. Lefering R, Zart M, Neugebauer EAM. Retrospective evaluation of the Simplified Therapeutic Intervention Scoring System (TISS-28) in a surgical intensive care unit. Int Care Med 2000; 26(12):1794-1802.

12. Castillo-Lorente E, River-Fernandez R, Rodriguez-Elvira M, VazquezMata G. TISS-76 and TISS-28: correlation of two therapeutic activity indices on a Spanish multicenter ICU database. Int Care Med 2000; 26(1): 57-61.
13. Pierin AMG, Padilha KG, Cruz DALM. Caracterização dos pacientes de duas Unidades de Terapia Intensiva (UTI): condições bio-sociais, processo de internação e intervenções terapêuticas. Rev Esc Enferm USP 1990; 24(3):371-388.

14. Paiva SAR, Matai O, Resende NO, Campana AO. Análise de uma população de doentes atendidos em unidade de terapia intensiva estudo observacional de sete anos (1992-1999). Rev Bras Terap Intens 2002; 14(2):73-80.

15. Moreira da Silva, Sousa RMC. Caracterização dos pacientes adultos e adolescentes das unidades de terapia intensiva do município de São Paulo. Rev Paul Enferm 2002; 21(1):50-7.

16. Ducci AJ, Padilha KG. Caracterização da gravidade de pacientes adultos internados em Unidade de Terapia Intensiva: Análise evolutiva segundo o Therapeutic Intervention Scoring System-28 (TISS-28). Rev Bras Terap Intens 2004; 16(2):22-27.

17. Associação de Medicina Intensiva Brasileira - AMIB. $1^{\circ}$ Anuário Brasileiro de Unidades de Terapia Intensiva; 1999-2000.

18. Associação de Medicina Intensiva Brasileira (AMIB). $2^{\circ}$ Anuário Brasileiro de Unidades de Terapia Intensiva; 2002/03.

19. Livianu J, Orlando JMC, Proença JO. Comparação de 3 índices de gravidade de pacientes de Unidade de Terapia Intensiva. Anais do VII Congresso de Terapia Intensiva Adulto e Pediátrico. Salvador; 1997.

20. Ferrero OG, Mateo ME, Marin VG, Salas CL. Niveles assistenciales em um servicio de medicina intensiva: Análisis de escalas de esfuerzo terapêutico y nível de gravidad. Enferm Intensiva 1999; 10(1):13-21.

21. Reis Miranda D, Ryan DW, Schaufeli WB, Fidle V. Organisation and management of intensive care: a prospective study in european countries. New York (USA): Springer; 1998.

22. Ministério da Saúde. Estabelecimentos de critérios de classificação para as Unidades de Tratamento Intensivo - UTI. Portaria $N^{\circ} 3432$; 1998. 